\title{
Chemical restraint of endangered mammals for conservation purposes: a practical primer
}

\author{
Steven A. Osofsky and Karen J. Hirsch
}

\begin{abstract}
Wild mammals sometimes need to be restrained for conservation purposes. Game rangers, wildlife managers, field biologists and wildlife veterinarians need to be fully conversant with a wide array of environmental and biological variables when using chemical restraint on wild mammals. In order to minimize risks to subject animals, they also need to be able to react appropriately using the correct equipment with sufficient skill to deal with anything that can go wrong. The importance of thorough background research and planning before going into the field cannot be over-
\end{abstract}

emphasized.The physiological results of immobilization drugs delivered by dart depend on dosages, the success of dart placement and drug delivery in good muscle, as well as the physiological state of the animals prior to and during anaesthetic induction. This paper describes the essential components of a planning and monitoring protocol for chemically restraining terrestrial mammals under field conditions.

Keywords Anaesthesia, conservation, chemical restraint, mammals, monitoring, safety.

\section{Introduction}

Conservation efforts focused on wild mammals and their ecosystems often rely on research methodologies that require the handling of subject animals. Perhaps the most common reason for handling mammals is for the purposes of marking or radiocollaring. Sound management may require a better understanding of a species's habitat requirements over the seasons, a knowledge of the factors affecting home range size, estimates of density under different ecological conditions, the unravelling of reproductive histories, etc. Of course, there are other reasons for handling wild mammals, ranging from the procurement of biomedical samples for research purposes to the translocation of the animals to another area, or even into captivity (Osofsky, 1994). Wildlife managers may elect to intervene when wild animals (particularly endangered species) are injured as a result of human activities (Osofsky, 1988, 1993). More often than not it is wildlife biologists who handle the highly endangered mammals of greatest concern and who accept responsibility for the inherent risks (Creel et al., 1997).

The majority of conservation practitioners do not have easy access to the veterinary literature. The

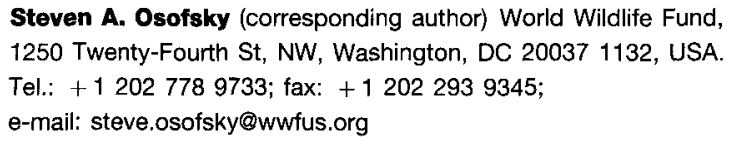

Steven A. Osofsky (corresponding author) World Wildlife Fund, 1250 Twenty-Fourth St, NW, Washington, DC 20037 1132, USA. Tel.: +1202 778 9733; fax: + 1202293 9345; e-mail: steve.osofsky@wwfus.org

Karen J. Hirsch 11697 Fox Glen Drive, Oakton, VA 22124, USA.

Received 4 March 1999. Accepted 1 July 1999 purpose of this paper is to provide them with information that veterinarians consider to be both practical and essential. This paper grew out of informal field notes that we developed over the years specifically to help biologists think through a component of their work (the capture of subject animals) that was clearly a means to a larger end (the successful completion of a study), and not something in which they necessarily wanted to invest a great deal of time. Users of this primer may find some components more useful than others, depending on their own level of field experience and the particular needs of their projects.

A variety of mechanisms contributes to the assessment of whether or not the potential benefits of a handling exercise exceed the potential costs, particularly with respect to an endangered species: institutional animal care and use committees, peer review of grant proposals, donor agency project proposal evaluation, animal welfare group influence, host-country wildlife policies and legislation, and even common sense. The purpose of this paper is not to examine philosophically when the handling of wild mammals is or is not justified (Rolston, 1992). We start at the point that a project is going to be carried out, when there is a reasonable consensus that the effort is justified as being in the best interest of conservation of the subject species. The inherent risks to the subject individual(s) need to be clearly understood and kept to a minimum.

\section{Methodology and discussion}

Once the decision to handle animals has been made, the next consideration is how best to do it. For most 
mammalian taxa, there is fairly broad consensus as to when mechanical capture (passive or active trapping) is best applied, and when chemical restraint is indicated as the primary capture method. That decision should be based on a thorough evaluation of the literature, along with appropriate consultation with people familiar with the species to be handled. For conservation projects that require the handling of mammals, chemical restraint is often indicated either to catch the animals or to handle them safely once captured by mechanical means.

Chemical restraint in its most basic form involves paralysing an animal without affecting its sensory perceptions. Mammalian capture by paralysis with drugs such as succinylcholine no longer has a place in wildlife conservation projects, given the safer and more humane pharmacological options now available. Immobilization via partial anaesthesia diminishes an animal's locomotor and sensory capabilities to a degree sufficient to allow capture and handling. Complete (general) anaesthesia yields an animal that is completely unconscious and, presumably, without pain perception. These pharmacologically induced states can be thought of as a gradient, albeit a somewhat irregular one, and the point attained is dependent on a wide array of factors. The most obvious of these are the types and dosages of drugs used and the species receiving them. The irregularities arise when all does not go as planned. Game rangers, wildlife managers, field biologists and wildlife veterinarians all have one thing in common when using chemical restraint on wild animals: they all need to be fully conversant with that wide array of factors, and to be able to apply the appropriate procedures using the right equipment with sufficient skill to deal with anything that can go wrong. Appendix A lists some of the basic factors that can affect the chemical capture of mammals via projectile syringe (dart). The importance of thorough background research and planning before going into the field cannot be overemphasized. The value of experience (and sometimes luck) should not be underestimated.

In order to minimize anaesthetic risk, we recommend familiarity with the basic facts, procedures, equipment and intervention skills outlined in Appendix B. It is neither possible nor desirable to summarize the entire realm of information available on veterinary anaesthetic monitoring and emergencies here. Currently, several types of wildlife capture workshops and short courses are available world-wide. In addition, national, provincial or state veterinary authorities can be helpful in identifying reputable operators, as well as those offering some form of officially recognized certification. A range of literature references should be consulted when a mammalian capture exercise is being planned (Harthoorn, 1976; Nielsen et al., 1982; Muir \& Hubbell, 1989, 1991; Clark \& Jessup, 1992; Fowler, 1993; McKenzie,
1993; Nielsen \& Beheler-Amass, 1995; Kreeger, 1996; Karesh et al., 1998; Sapolsky \& Share, 1998).

Adherence to even the most comprehensive set of guidelines cannot completely prevent the possibility of immobilization mishaps. Our hope here, however, is to reduce their incidence and to lessen their severity when they do occur. In addition, in our experience, it is quite feasible to pack efficiently the necessary project-specific drugs and equipment in backpacks and tackle boxes, even when working from a helicopter.

A successful chemical restraint exercise is not complete until the subject animal is fully (both physically and mentally) recovered and (for field research) back in its natural environment. The application of appropriate pharmacological principles, with an emphasis on drug reversibility, will minimize the chances that the animal will be at a competitive disadvantage, be disoriented and thus subject to injury, or be 'easy prey' following its release. Residual sedation and renarcotization must be avoided in the field.

\section{Conclusions}

The application of basic veterinary knowledge by welltrained personnel can make a substantial contribution to both animal and human safety during capture exercises. Regardless of the academic qualifications of the scientists and managers working on a particular field project, teams truly qualified to handle endangered wild mammals should include appropriate taxonspecific expertise in wildlife anaesthesia-not just as consultants during planning, but on the ground. The interface between wildlife conservation and animal welfare, like the interface between population-level and individual animal medicine, is an intricate one that conservation professionals must continue to strive to understand if we are to maximize our contribution to the maintenance of free-ranging wildlife for the future.

\section{References}

Andrews, E.J., Bennett, B.T., Clark, J.D., Houpt, K.A., Pascoe, P.J., Robinson, G.W. \& Boyce, J.R. (1993) 1993 report of the AVMA panel on euthanasia. Journal of the American Veterinary Medical Association, 202, 229-249.

Bistner, S.I. \& Ford, R.B. (eds) (1995) Kirk and Bistner's Handbook of Veterinary Procedures and Emergency Treatment, 6th edn. W. B. Saunders Company, Philadelphia.

Bush, M. (1992) Remote drug delivery systems. Journal of Zoo and Wildife Medicine, 23, 159-180.

Clark, R.K. \& Jessup, D.A. (eds) (1992) Wildlife Restraint Series. International Wildlife Veterinary Services, Salinas.

Creel, S., Creel, N.M. \& Monfort, S.L. (1997) Radiocollaring and stress hormones in African wild dogs. Conservation Biology, 11, 544-548. 
Ebedes, H. (1992) The Use of Tranquillizers in Wildlife. Department of Agricultural Development, Pretoria.

Fowler, M.E. (ed.) (1993) Zoo and Wild Animal Medicine, Current Therapy 3. W.B. Saunders Company, Philadelphia.

Harthoorn, A.M. (1976) The Chemical Capture of Animals. Bailliere Tindall, London.

Karesh, W.B., Wallace, R.B., Painter, R.L.E., Rumiz, D., Braselton, W.E., Dierenfeld, E.S. \& Puche, H. (1998) Immobilization and health assessment of free-ranging black spider monkeys (Ateles paniscus chamek). American Journal of Primatology, 44, 107-123.

Kreeger, T.J. (1996) Handbook of Wildlife Chemical Immobilization. International Wildlife Veterinary Services, Laramie.

McKenzie, A.A. (ed.) (1993) The Capture and Care Manual: Capture, Care, Accommodation and Transportation of Wild African Animals. Wildlife Decision Support Services and the South African Veterinary Foundation, Pretoria.

Muir, W.W. \& Hubbell, J.A. (eds) (1989) Handbook of Veterinary Anesthesia. C.V. Mosby Company, St Louis.

Muir, W.W. \& Hubbell, J.A. (eds) (1991) Equine Anesthesia: Monitoring and Emergency Therapy. Mosby-Year Book, St Louis.

Nielsen, L. \& Beheler-Amass, K. (1995) Chemical Immobilization of Animals. Safe-Capture International, Mt Horeb.

Nielsen, L., Haigh, J.C. \& Fowler, M.E. (eds) (1982) Chemical Immobilization of North American Wildlife. Wisconsin Humane Society, Milwaukee.

Osofsky, S.A. (1988) Panther diary. Natural History, 97, $50-54$.

Osofsky, S.A. (1993) Ethical triage in free-ranging wildlife in Botswana. World Association of Wildlife Veterinarians Newsletter, 13, 8-9.

Osofsky, S.A. (1994) Botswana's wildlife resource: a veterinary perspective. Journal of the American Veterinary Medical Association, 205, 1381-1385.
Rolston III, H. (1992) Ethical responsibilities toward wildlife Journal of the American Veterinary Medical Association, 200, $618-622$.

Sapolsky, R.M. \& Share, L.J. (1998) Darting terrestrial primates in the wild: a primer. American Journal of Primatology, 44, 155-167.

\section{Biographical sketches}

Dr Steven Osofsky is a wildlife veterinarian with a longstanding interest in international wildlife conservation. His first experience in Africa was as a Harvard University Travelling Fellow in 1984-85, when he had the opportunity to observe wildlife species in East and Central Africa while examining conservation problems from a variety of perspectives. He subsequently attended veterinary school at Cornell University, receiving his Doctor of Veterinary Medicine in 1989. He served the Government of Botswana's Department of Wildlife and National Parks (DWNP) as the country's first Wildlife Veterinary Officer from 1992 to 1994 . He has also worked as a zoological veterinarian, as a Biodiversity Program Specialist for the US Agency for International Development (USAID), and is currently the Senior Program Officer for WWF-US's Species Conservation Program, working on African and Asian efforts.

Dr Karen Hirsch is also a veterinarian (Cornell University, 1989) with interests in the interface between wildlife conservation and veterinary ethics. She served as a volunteer field and laboratory assistant in Botswana's Wildlife Veterinary Unit, and helped with training and capacity building in the DWNP.

\section{Appendix A: Some basic factors affecting the process of chemical capture of mammals via projectile syringe (dart)}

Please note that many of these factors can also affect the subject's successful release back into the wild at the conclusion of the exercise. There is some obvious overlap when attempting to categorize these factors.

\section{Behavioural}

Typical flight distance of species.

Social structure of target species.

Status of subject within social structure.

Presence of conspecifics, and reactions thereof.

Presence of other species, and reactions thereof.

\section{Physiological}

Estimated versus actual weight of the subject. Actual health of subject.
Actual age of subject.

Nutritional status of subject.

Sex, reproductive status of subject (NB: females and their young must be reunited safely for an exercise to be considered successful).

Time of the subject's most recent meal.

Level of subject's arousal/stress prior to darting.

Level of subject's arousal/stress following darting.

Seasonal variations, depending on species, drugs used.

Anatomical site of dart injection (depends on species, size of subject). Darts are dangerous if targets are missed or if capture equipment is used incorrectly. Hindquarter muscles are the safest targets in terrestrial mammals, and allow for rapid drug absorption. Blubber (as in pinnipeds) should be avoided.

Shock (hypotensive, hypovolaemic or haemorrhagic).

Hypothermia.

Hyperthermia.

Events or clinical signs indicating that capture myopathy (exertional rhabdomyolysis) is likely.

Position of subject during recumbency (see Appendix B). Bloat (which can compromise respiration), regurgitation or 
vomiting (which can lead to asphyxiation or aspiration of ingesta/digesta).

Traumatic injuries to subject animal during capture.

\section{Pharmacological}

Type(s) of drug(s) selected (cyclohexamines, opiates, opioids, benzodiazepines, alpha-2-adrenergic agonists,

phenothiazines, butyrophenones, anticholinergics, hyaluronidase).

Drug dosages selected.

Species-specific reactions to different capture drugs.

Undesirable effects of drugs used (particularly neurological including, for example, seizures; cardiovascular; respiratory), and dangers thereof (see Appendix B).

Availability of antagonists for restraint drug(s) selected.

Need for additional drug administration if capture is not successful, or during handling.

\section{Environmental}

Ambient conditions (temperature, humidity, wind).

Terrain (open, wooded, mountainous, riverine, presence of standing water, cliffs or ravines).

Amount of daylight remaining.

\section{Equipment-related}

Type of projector and dart used (a wide variety is available, each with particular strengths and weaknesses; Bush, 1992).

Degree of success of intramuscular drug delivery.

Degree of success of drug volume delivery.

Reliability of communications and transport.

\section{Personnel}

Presence of sufficient number of adequately trained personnel.

Disturbances (such as noisy personnel) at all stages of the exercise.

\section{Appendix B: Field anaesthesia monitoring: basic facts, procedures, equipment and intervention skills}

Please note that when in trouble or in doubt, administer the appropriate antagonist intravenously (or intramuscularly if venipuncture proves difficult) to arouse the animal as quickly as possible. Most acute anaesthetic complications will be alleviated by this action. The procedure can be rescheduled for another day.

\section{Duration}

Minimizing the time the animal is anaesthetized is perhaps the single most effective way to prevent the majority of anaesthetic complications.

\section{Body position}

Sternal, semi-sternal, left lateral, right lateral, etc. Different taxonomic groups have different requirements and limitations to preclude cardiovascular and/or respiratory compromise, and to prevent bloat, aspiration, neuropraxia and/or compartmental syndrome. Learn the recommended recumbent position for the target species as part of the capture planning process. Level, soft surfaces are ideal

\section{Respiration}

Know what is normal for the species you are handling/anaesthetic protocol you are using.

Assisted/emergency ventilation if needed: intubate animal (see basic equipment list below).

Carnivore example: one breath every $4 \mathrm{~s}$ (duration of inspiration: expiration, ratio 1:3). Equid example: one breath every 6-10 s (duration of inspiration: expiration, ratio $1: 2$ ). (Blood gases/pulse oximetry helpful in guiding this. A compact, portable pulse oximeter is strongly recommended to provide immediate feedback on oxygenation trends during uncomplicated immobilization procedures as well as during emergencies.) Monitor the chest wall to determine the appropriate volume of air or oxygen to administer. Chest excursion at maximum inhalation should be slightly more than, or equal to, the expansion seen in an awake animal. Carried out improperly, artificial ventilation can do more harm than good. See notes on demand valve and Ambu bag below.

A stethoscope can aid in the diagnosis of pulmonary problems (for example, pneumothorax) that can be life-threatening. See notes on cardiothoracic auscultation below.

\section{Pulse pressure/rate/rhythm}

Know what is normal for the species you are handling/anaesthetic protocol you are using.

Depending on species, use femoral artery (inner thigh), carotid artery (neck), facial artery (mandible), median caudal artery (under base of tail), dorsal metatarsal artery (distal hind limb), auricular artery (ear), lingual artery (underside of tongue), etc, to check pulse.

Intravenous fluids and/or pharmacological intervention may be indicated if the pulse pressure is weak even if the heart rate is normal. Irregular pulses coincide with cardiac arrhythmias (below).

\section{Cardiothoracic auscultation}

Always use a stethoscope and know what is normal for the species being handled/anaesthetic protocol being used. Early detection of arrhythmias, which are often the result of myocardial hypoxia, can be life-saving. Pulmonary problems can be diagnosed quickly. For example, pneumothorax can result from a misplaced dart and can be life-threatening if not treated. 


\section{Mucous membrane colour}

Unpigmented gums should appear pink. Purple-greyish mucous membranes indicate cyanosis (poor oxygenation). This should be treated as a respiratory emergency. Very pale mucous membranes may indicate a circulatory (cardiac) problem. Check the cardiac parameters (such as pulse rate and strength) and decide if action is indicated. Note that clinically significant low arterial blood oxygen saturation can be detected much earlier in its progression using a pulse oximeter than by visual examination of the mucous membranes.

\section{Capillary refill time (CRT)}

Press down on the gums momentarily to occlude blood flow, then release pressure, observing time taken for pink colour to return. Normal CRT $\leq 2 \mathrm{~s}$. CRT $>2 \mathrm{~s}$ under anaesthesia is suggestive of poor peripheral perfusion, low blood pressure, poor cardiac function. Check the cardiac parameters (pulse rate and pressure) and decide if action is indicated. Note that, overall, CRT is a very crude indicator of patient status, and monitoring of the other parameters described should lead to earlier detection of anaesthetic complications.

\section{Temperature}

One needs to know how the animal is faring with regard to perhaps the most common capture-related problem: overheating. Temperature monitoring is also needed to provide feedback on cooling attempts. A digital thermometer with a soft wire rectal probe placed deep into the rectal cavity is more likely to give accurate readings than a more superficially placed human or large-animal digital (avoid glass) rectal thermometer. Continuous readings help to highlight trends. Uncomplicated mammalian chemical immobilization is generally associated with temperatures in the $100-103^{\circ} \mathrm{F}$ $\left(37.8-39.4^{\circ} \mathrm{C}\right)$ range. Conservatively, use $104^{\circ} \mathrm{F}\left(40^{\circ} \mathrm{C}\right)$ as the point at which to begin actively cooling the animal. Depending on species, drug protocol and environment, $106^{\circ} \mathrm{F}\left(41.1^{\circ} \mathrm{C}\right)$ should be treated as a hyperthermic emergency. Hypothermia can be a serious problem (usually but not always in cool environments) and requires careful treatment. Consult reference texts, such as Kreeger (1996).

\section{Palpebral response}

The palpebral response can give a relative indication of depth of anaesthesia. Gently tap the corner of the eye without touching the cornea: a blink response correlates with a light plane of anaesthesia; the absence of a blink response suggests a deeper plane of anaesthesia. However, reflexes alone should not be relied upon as indicators of anaesthetic depth.

\section{Cardiopulmonary resuscitation (CPR)}

If necessary, CPR can be administered but is more practical for small to medium-sized carnivores than for large ungulates:

Airway: intubate and inflate cuff.

Breathing: start giving breaths (see notes on respiration above).

Circulation: 60-80 cardiac compressions per minute. Five compressions to one ventilation-keep rhythm going and do not stop during the ventilatory event.

Consult a range of veterinary references for CPR details and for pharmacological intervention protocols for cardiac arrest (Muir \& Hubbell, 1989, 1991; Bistner \& Ford, 1995; Kreeger, 1996).

\section{Basic emergency/useful drugs}

Yohimbine, atipamezole, epinephrine, atropine, doxapram, naltrexone, naloxone, nalorphine, diprenorphine, diazepam, midazolam, dexamethasone sodium phosphate, prednisolone sodium succinate, methyprednisolone sodium succinate, sodium bicarbonate, lidocaine, calcium chloride or gluconate, procainamide, ketamine, dopamine, furosemide, aminophylline, diphenhydramine, heparin, tetanus toxoid and antitoxin, antivenin, 50 per cent dextrose solution, isotonic saline solution, lactated Ringer's solution, hypertonic saline solution, colloidal plasma substitute, vitamin E, selenium, B-complex vitamins, short- and long-acting antibiotics, topical disinfectants, eyewash, antibiotic infusion preparations for dart site treatment, hydrogen peroxide for blood stain removal, insect repellents, euthanasia solution, etc.

Carry an assortment of syringes, needles, intravenous and intraosseous catheters, fluid administration sets, tourniquets, rubber gloves. The correct placement of catheters takes practice. All emergency protocols must be clearly understood by the responsible personnel before the capture exercise begins. The indications and contraindications of each drug on hand must be understood. For projects involving translocation or the holding of animals, specialized short- and/or long-acting tranquillizers (neuroleptics) may be indicated (Ebedes, 1992).

Prior to darting, appropriate amounts of the necessary anaesthetic antagonist agent(s) should be drawn into a labelled syringe (or syringes) with needle(s) of appropriate gauge and length for the subject species to preclude any delays if reversal is needed in an anaesthetic emergency.

\section{Subsequent dosing after partial or questionable dart} injections

The handling of this situation will vary with experience, species, drug protocol and environment. Generally, if the animal shows minimal effects after $15 \mathrm{~min}$, repeat initial cocktail dosages. The intravenous administration of supplemental ketamine may be needed when mammalian subjects can be caught but are not adequately anaesthetized for safe handling. Sometimes a partially drugged animal needs to be darted with the appropriate antagonist with a drop-out dart if further attempts at 
capture appear too risky. Experience and discretion influence this decision.

\section{Human safety}

Wildlife work is often carried out in remote areas and a capture team must have contingency plans to deal with human injuries, including exposure to dangerous capture drugs-particularly narcotics. Research of current literature and consultation with appropriate experts on the dangers of specific drugs and equipment should be part of the capture planning process, culminating in written emergency response protocols, self-contained human emergency kits including antagonists of narcotics, and appropriate training for all members of the capture team (including training in basic human CPR). Local medical facilities should be alerted when exercises involving dangerous drugs are being scheduled. Often, these facilities need orientation on the special hazards posed by capture drugs unfamiliar to human medical practice. National and local legislation pertaining to animal drugs, and liability issues regarding human (and animal) welfare should be clearly understood before a project is initiated.

\section{Record keeping}

Written records are essential to developing good anaesthesia protocols. Keep track of environmental conditions, the subject's responses over time to the doses given, vital sign trends under anaesthesia, time for the reversal agent to take effect, and time to full recovery. This type of information is best recorded on a standard form and helps to guide dose modifications for subsequent anaesthesia involving that particular subject or species. Checking and recording vital signs at least every $3-5 \mathrm{~min}$ reduces the chances of irreversible anaesthetic complications.

\section{Basic equipment (in addition to that mentioned above)}

Permanent markers to label drug syringes, sample collection tubes, vials, plastic bags, swabs, slides, etc. Biomedical sampling and physical examination opportunities should be planned in advance and maximized, especially for endangered species.

Emergency (reflecting) blanket for hypothermia cases. Can also be used to provide shade for anaesthetized subjects.

Ropes to restrain and secure larger animals during capture, reducing chances of injury to both subject and personnel.

Rubber hose sections to place on horns of antelopes etc. to protect personnel.

Sterile ophthalmic ointment, placed gently in each eye to protect the cornea. The protective blink reflex can be compromised by capture drugs. Do not touch the tip of the ointment tube against the cornea itself.

Blindfold to protect the eyes and keep the animal calm if anaesthetic plane is light.

Cotton earplugs for very sensitive species or special situations (remove before release).
Portable fur-clippers, to facilitate catheter placement and suturing of lacerations, etc.

Tape (masking or bandage), absorbable suture, and/or ethyl cyanoacrylate-type glue to secure catheters when large volumes of intravenous fluids are needed.

Basic pack of sterile surgical instruments and gloves, along with absorbable suture materials of assorted sizes to address lacerations or other injuries sustained during capture.

Water (ideally at least $20 \mathrm{~L}$ ) for topical cooling if needed. Pinnipeds have special needs for the topical application of water and/or ice.

Chemical hot and cold packs, intravenous fluid lines.

Isopropyl alcohol in spray bottle as disinfectant and for topical cooling used on neck, chest, armpits, ears, inner thighs (avoid eyes).

Endotracheal tubes in various sizes for intubation.

Sterile water-soluble lubricant to ease the passage of endotracheal tubes, for example.

Stylette to stiffen endotracheal tubes and ease insertion.

Cuff syringe to inflate endotracheal tube cuff (which provides a seal, decreasing risk of aspiration pneumonia in case of vomiting or regurgitation).

Laryngoscope (various blade sizes available) to move the tongue and help view the larynx when inserting endotracheal tubes. A tongue depressor and flashlight will work in an emergency in small animals.

Mouth gag to keep the mouth open during intubation.

Gauze roll to secure the endotracheal tube to the mandible, maxilla or head (behind the ears). Use quick release knots. Tape can also be used, although it can be more difficult to remove. Gauze pads are useful for holding on to a slippery tongue during intubation, applying to venipuncture sites, etc.

Sponge forceps and gauze pads to remove saliva, food, debris from the oropharynx.

Portable oxygen tank with regulator.

Demand valve with control button and hose for use on the oxygen tank regulator to allow artificial ventilation (described above). An Ambu bag can also be used but generally not for animals much heavier than an adult human. An Ambu bag can be used to ventilate a subject, even without supplementary oxygen.

Stomach tubes for relieving bloat in ruminants, etc. A rumen trocar/cannula should be used only as a last resort in a life-threatening situation: hypodermic needles are slower in relieving refractory bloat but are less traumatic and less likely to lead to infection. 'Stomach' tubes can also be used to administer cool (warm) water enemas in hyperthermia (hypothermia) cases. A Jorgenson-type pump, when used carefully, can expedite this process.

Bucket.

(Trocar) chest tubes, connecters, 60-mL syringes, three-way stopcocks, Heimlich valve: for pneumothorax cases.

Stretcher for carrying large animals.

Ground tarpaulin.

Bandage, support wrap and cast materials.

Saws and hedge clippers to free animals entangled in vegetation during capture.

Toolbox and spares for vehicle, communication equipment. 
Post-mortem examination and sample collection supplies. If an animal dies, it is important to determine exactly what went wrong in order to avoid repetition of mistakes. Sometimes an animal is irreparably harmed during an exercise and in such rare cases a humane method of euthanasia should be employed (Muir \& Hubbell, 1989; Andrews et al., 1993). This is an extremely difficult situation, particularly for endangered species, and is best avoided by using 'best practices' at all times. 